\title{
Research Review of Principles and Methods for Ultrasonic Measurement of Axial Stress in Bolts
}

Qinxue Pan ${ }^{1,2^{*}}$ (D) Ruipeng Pan ${ }^{1,2}$, Chang Shao ${ }^{1,2}$, Meile Chang ${ }^{1,2}$ and Xiaoyu Xu $\mathbf{u}^{1,2}$

\begin{abstract}
Bolts are important fasteners indispensable in the manufacturing field for their advantages, which include convenient assembly and disassembly, easy maintenance, refastenability to prevent looseness, and the avoidance of a phase change in the connected material composition. The precise control of the tightening force in bolts is closely related to the safety and reliability of the connected equipment or structure. Although there are many methods for estimating the tightening force applied to a bolt during assembly, poor accuracy in controlling the preload during the tightening process and a lack of monitoring to determine the residual axial force in service remain issues in evaluating the safety of bolted assemblies. As a nondestructive testing technology, ultrasonic measurement can be applied to successfully address these issues. In order to help researchers understand the theoretical basis and technological development in this field and to equip them to conduct further in-depth research, in this review, the basic knowledge describing the state of stress and deformation of bolts, as well as conventional testing methods are summarized and analyzed. Then, through a review of recent research of the ultrasonic measurement of the axial stress in bolts, the influence of the effective stressed length and temperature are analyzed and proposed methods of calibration and compensation are reviewed. In order to avoid coupling errors caused by traditional piezoelectric transducers, two newly proposed ultrasonic coupling technologies, the electromagnetic acoustic transducer (EMAT) and the permanent mounted transducer system (PMTS), are reviewed. Finally, the new direction of research of the detection of residual axial stress in in-service bolts that have been assembled to yield is discussed.
\end{abstract}

Keywords: Bolted connections, Axial stress, Ultrasonic waves, Influencing factors, Coupling techniques

\section{Introduction}

Bolts are indispensable fasteners in the manufacturing and national defense industries and are critical components of important infrastructure, such as aerospace, vehicle, ship, railway, bridge, and building facilities. Each application of bolts has its own construction standard, such as GB50205 "Code for Acceptance of Construction Quality of Steel Structures" in the structural engineering field and TBJ214 "Construction Regulations on High Strength Bolt Connection of Railway

\footnotetext{
*Correspondence: panqx@bit.edu.cn

'School of Mechanical Engineering, Beijing Institute of Technology,

Beijing 100081, China

Full list of author information is available at the end of the article
}

Steel Bridge" in the railways field [1]. The fundamental purpose of these standards is to ensure reliable and durable connection between parts to meet the requirements of structural integrity and stability. The quality of a bolted connection depends largely on the control of the preload in the bolt. This preload depends first and foremost on the strength of the bolt: the greater the strength, the greater the potential preload and the better the resistance of the bolt to loosening and fatigue. The performance of a bolt when tensioned to yield is best [2]. Under these circumstances, the upper limit of the preload is determined by the strength of the bolt, nut, and connected parts. An excessive preload will cause severe plastic deformation of the bolt which could lead to failure due to fatigue or fracture. 
Accordingly, the preload should usually be less than $80 \%$ of the yield strength of the material, or within $35 \%$ to $70 \%$ of the breaking force. The lower limit of the preload is determined by the required function of the connected parts, as insufficient preload will cause vibration, slack, and slip of structural connections that can damage the integrity of the structure and affect the normal operation of any bolt-assembled equipment [3]. Additionally, due to the influence of applied external load and changes in ambient temperature during service, a bolted connection can gradually loose tension, but this loss is not easy to detect. In the absence of a system for post-installation monitoring of bolts, the adverse effects of loosening bolts will gradually expand and can result in serious consequences. Therefore, a method for the accurate control of bolt preload during tightening and in-situ online monitoring of the residual axial force during the service life of the connection is critical to ensure the stability and reliability of boltassembled structures.

At present, bolt preload is usually controlled by the torque method or the turn-of-the-nut method in engineering applications. However, it is difficult to achieve high precision using these methods because of variations in actual working conditions, low accuracy of construction equipment, and lack of experience among workers. To address these issues, ultrasonic measurements have been used for detection of the axial stress in bolts since the 1980s. As an emerging nondestructive measurement technology, the ultrasonic measurement technique has been favored by researchers both domestically in China and abroad for its advantages of high precision, excellent real-time performance, and strong sensing penetration. In order to help researchers understand the basic theory and technical development of ultrasonic measurement, promote further development of ultrasonic technology, and provide methods for rapid and accurate evaluation of the working state of tensioned bolts, this paper reviews the research progress of this technology in the following sections. Section 2 briefly discusses the stress and deformation states of bolts during assembly and service to provide researchers with an overall understanding of the object to be tested. Section 3 summarizes the measurement methods currently used and discusses the drawbacks of these methods in engineering applications. Section 4 reviews research of the ultrasonic measurement of the axial force in bolts conducted domestically and abroad in recent years. On this basis, Section 5 analyzes the main influencing factors and corresponding methods of calibration and compensation. In Section 6, challenges and opportunities for applying ultrasonic testing technology to determine the axial stress in bolts in
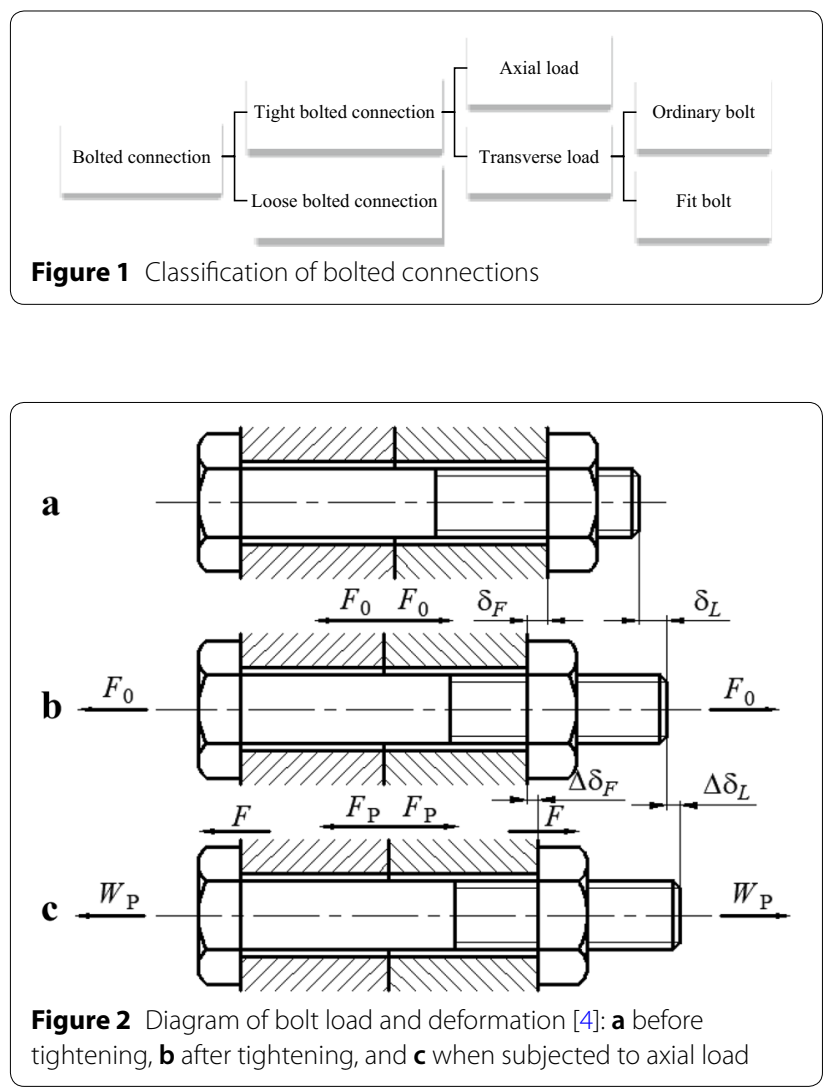

industrial applications and academic research are discussed. Finally, conclusions are drawn in Section 7.

\section{Analysis of Stress and Deformation}

Bolted connections are classified as shown in Figure 1 according to the way the bolt load is applied to the connection. Bolted connections are first divided into tight and loose bolted connections based on whether the bolts must be tightened during assembly. In addition to the preload, a tight bolted connection can resist both transverse load and axial load according to the service requirements. However, loose bolted connections can only resist a single direction working load and are mainly used in applications such as hooks that do not need to be tightened.

The actual bolt tightening process is shown in Figure 2, where Figure 2(a) shows the state of a bolt before tightening. The application of the tensile preload $F_{0}$ then stretches the bolt by $\delta_{L}$. At this time, the connected parts are compressed by $\delta_{F}$, as shown in Figure 2(b). For an ordinary bolted connection subjected to a transverse load, the bolt shank and the connected plies have a clearance fit. After tightening, a friction force is generated between the connected plies to prevent them from sliding relative to each other. For a fit bolt, there is no clearance 
between the bolt shank and the hole wall, so the transverse load is mainly resisted by the contact between the bolt and the hole walls of the connected plies, and by the shear deformation of the bolt shank [4].

When a fastened bolt is subjected to an axial load, the deformations of the bolt and the connected plies are as shown in Figure 2(c). The axial load $F$ increases the elongation of the bolt to $\delta_{L}+\Delta \delta_{L}$, while the corresponding tensile force is the total axial force in the bolt $W_{\mathrm{P}}$. With the application of axial load, the connected plies pull apart with the elongation of the bolt by $\Delta \delta_{F}$, and the remaining pressure is the residual preload $F_{\mathrm{P}}$. The stress states of the bolt and the connected plies are shown in Figure 3. The straight lines DB and FA indicate the relationships between the axial deformation and the axial force of the bolt and the connected plies, respectively. Before tightening, the stress states of bolt and connected plies are point $\mathrm{D}$ and $\mathrm{F}$, respectively. When the preload is applied, point A is the stress state of the bolt and the connected plies. After an axial working load is applied, the stress state of bolt is point $B$, while the stress state of connected plies returns to point $C$. Therefore, the total axial force in the bolt $W_{\mathrm{P}}$ is the sum of the working load $F$ and the residual preload $F_{\mathrm{P}}$, as shown in Figure 3. The main purpose of ultrasonic measurement of the axial force in bolts is to accurately control the preload $F_{0}$ applied during assembly and to provide real-time monitoring of the total bolt axial force $W_{\mathrm{P}}$ under service.

\section{Conventional Measurement Methods}

Manual torque wrenches or pneumatic, hydraulic, or electric wrenches are commonly used to indirectly control bolt preload through the tightening torque. This method is simple in operation and low in cost, but is not very precise. Figure 4 depicts the distribution of torque consumption during the bolt tightening process $[6$, 7]. Most of the torque is absorbed by the need to overcome the friction between the contact surfaces of the

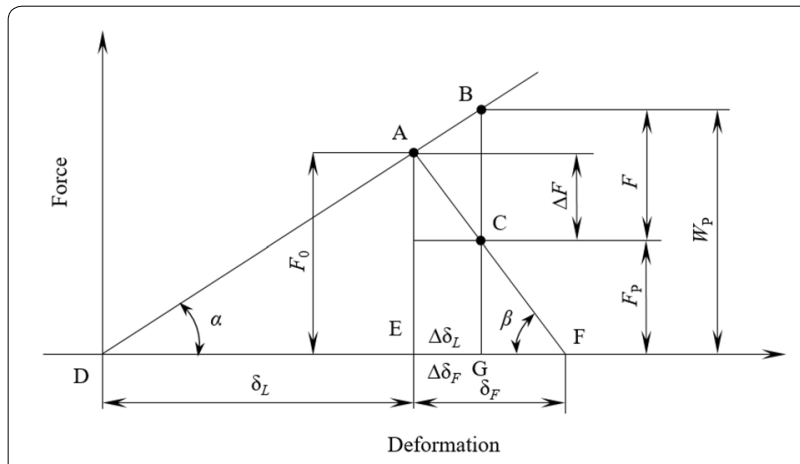

Figure 3 Relationship between bolt load and deformation [5]

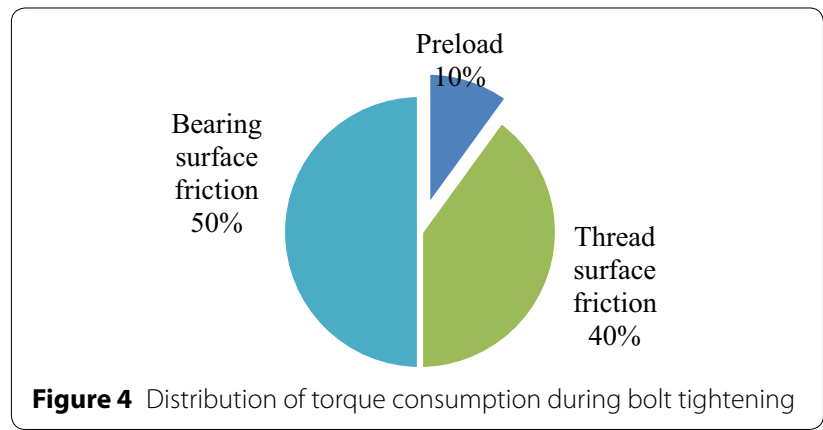

bolted connection, and only about $10 \%$ of the torque is converted into axial preload. Additionally, the friction coefficients of the thread surfaces and the nut bearing surfaces are quite variable, leading to significant uncertainty in the preload obtained by the torque method. Indeed, when applying the same torque to the same bolt under the same working conditions, the induced preload can vary by as much as $40 \%$ [8]. Furthermore, local areas such as the thread root usually exhibit large elastoplastic deformation, which can also have a negative effect on the accuracy of the torque method. Therefore, the torque method is typically only used in non-critical construction sites where accuracy requirements are not stringent.

The turn-of-the-nut method is a rotation control method that ensures the desired preload by rotating the nut by a certain angle. Under this method, the actual tightening process consists of the four stages shown in the preload-rotation angle curve of Figure 5: nut idling, tight contact between the nut and the connected plies,

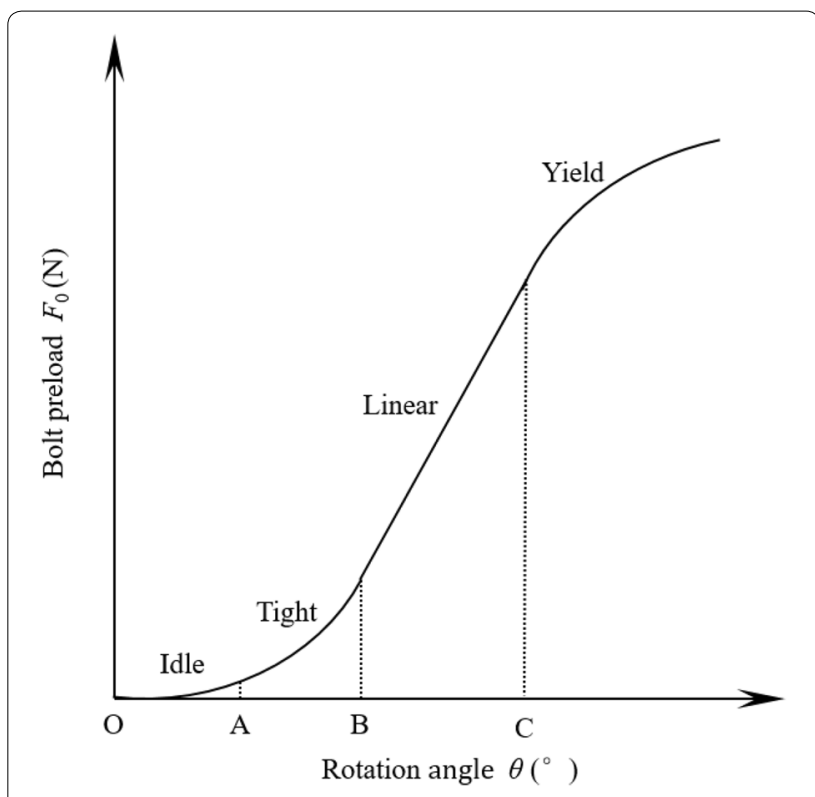

Figure 5 Relationship between bolt preload and rotation angle [9] 
linear increase in the preload, and bolt yield [2]. The torque/rotation method is more commonly adopted in engineering applications. Under this method, the bolt is first tightened to a certain torque value (beyond Point B in Figure 5) about $40 \%-60 \%$ of the full tightening torque. Then the turn-of-the-nut method is used to tighten the bolt to the required value. This method avoids the influence of friction on the axial clamping force and provides a higher precision than the torque method, but it is difficult to accurately determine the starting point of the angle measurement. During the tightening stage, different surface conditions can lead to different connection states between different plies under the same tightening torque [9]. Therefore, it can be difficult for erectors to determine when the nut has been sufficiently rotated to enter the linear phase shown in Figure 5.

The yield point method tightens the bolt all the way to its yield strength based on the relationship between the applied torque and the rotation angle. Using this method, it is necessary to monitor the torque-nut rotation angle curve in real time during tightening. When the slope of the curve drops below a certain value, generally defined as one-half of its maximum, the yield point is considered reached and the tightening is stopped [10], as shown in Figure 6 . This method is able to fully utilize the bearing capacity of the material and its tightening precision is very high, but it must first be calibrated to the fastener to establish the yield point and the distribution of the torque rate with respect to the applied rotation angle [10].

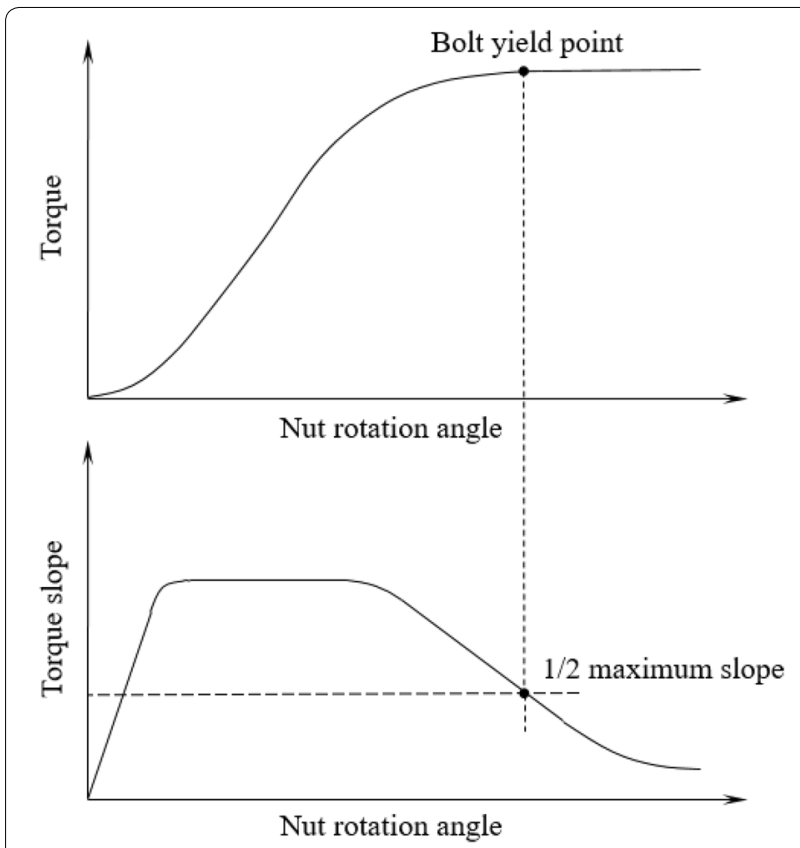

Figure 6 Relationship between torque and nut rotation angle [10]
The electric resistance strain gauge method utilizes the surface strain in a bolt to obtain its axial stress. A resistance strain gauge is accordingly attached within the clamping section of the bolt shank as shown in Figure 7. The change in the resistance of the strain gauge from before to after tightening is then measured by the resistance strain gage to indirectly obtain the axial stress in the bolt. The accuracy of this method is higher than that of the torque method, but it is necessary to attach a strain gauge to the surface of every bolt, which can be time consuming, difficult to do, and provides poor stability in application. Furthermore, only the stress on the surface of the bolt can be measured, and local stress concentrations after tightening can induce shear strain on the surface of the bolt, which can have an adverse impact on the measurement [11]. Accordingly, this method is only suitable for small batch detection and is gradually being replaced by other methods (Figure 7).

For sufficiently large-diameter bolts, the elongation of the bolt can be measured through a center hole to determine the applied preload. This method can eliminate the influence of the friction coefficient, contact deformation of the bolt itself, and deformation of the connected plies [5]. However, the actual service environment of most bolts is generally poor and the available space during assembly is small, which make it difficult to physically measure the elongation of bolts. Additionally, there is no convenient high-precision elongation measuring device and so it is currently difficult to meet the rapid assembly requirements for connections containing large quantities of bolts. This method too, then, can only be used for small-batch detection where strict accuracy is required.

A comparison of the bolt tension confirmation methods discussed in this section is provided in Table 1 . These methods are all applicable to the control of bolt preload and all have limited application to the detection of changes in the residual axial force in bolts in service. As a nondestructive and high-precision testing technology, the ultrasonic method can eliminate the influence of the friction coefficient on the measurement of torque and provide both online preload measurement during the assembly process and real-time monitoring of the change in residual axial force during service. Combined with computer technology and connected with convenient

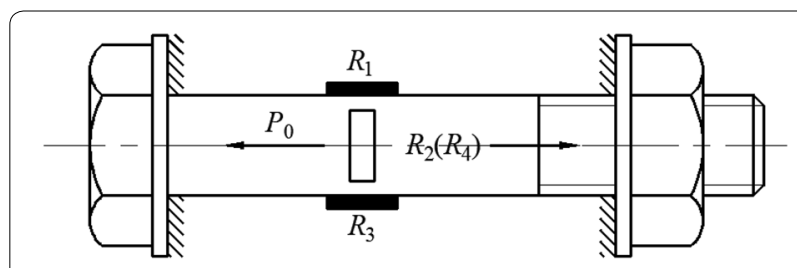

Figure 7 Resistance strain gauge detection assembly [1] 
Table 1 Comparison of conventional bolt tension confirmation methods $[1,11]$

\begin{tabular}{llll}
\hline Method & Measurement & Accuracy & Complexity \\
\hline Torque & Torque & $\pm 25 \%$ & Ordinary \\
Turn-of-the-nut & Torque + nut rotation angle & $\pm 15 \%$ & Slight \\
Yield point & Torque rate & $\pm 8 \%$ & Slight \\
$\begin{array}{l}\text { Electric resist- } \\
\text { ance strain }\end{array}$ & Resistance of strain gauge & - & Extreme \\
$\begin{array}{l}\text { gauge } \\
\text { Elongation }\end{array}$ & Elongation of bolts & $\pm 5 \%$ & \\
\hline
\end{tabular}

cabling that is easy to maintain, one or more sets of bolts can be measured simultaneously using the ultrasonic method.

\section{Ultrasonic Measurement Methods Based on Acoustoelasticity Theory}

Ultrasonic pulse-echo technology based on acoustoelasticity theory is widely used to measure the axial stress in bolts. Acoustoelasticity theory describes the relationship between the velocity of elastic wave propagation in a stressed solid material and not only the second-order elastic constants and density of the material, but also the high-order elastic constants and stress [12]. The relationship between the stress $\sigma$ and the velocity of longitudinal wave propagation along the direction of stress $v_{\sigma}^{\mathrm{L}}$ is [12]:

$$
\rho_{0} v_{\sigma}^{\mathrm{L}^{2}}=\lambda+2 \mu+\frac{\sigma}{3 K_{0}}\left[\frac{\lambda+\mu}{m}(4 \lambda+10 \mu+4 m)+\lambda+2 l\right],
$$

where $\rho_{0}$ is the density of the solid before deformation; $\lambda$ and $\mu$ are second-order Lame's constants; $l$ and $m$ are third-order Murnaghan's constants; and $K_{0}$ is the bulk modulus of the material under zero stress.

The relationship between the stress $\sigma$ and the velocity of a transverse wave whose direction of propagation is along the direction of stress and whose direction of polarization is perpendicular to the direction of stress $v_{\sigma}^{\mathrm{T}}$ is [12]:

$$
\rho_{0} v_{\sigma}^{\mathrm{T}^{2}}=\mu+\frac{\sigma}{3 K_{0}}\left[\frac{\lambda n}{4 \mu}+4 \lambda+4 \mu+m\right],
$$

where $n$ is the third-order Murnaghan's constant.

The applied axial stress causes the length of the bolt and the velocity of the ultrasonic waves to change at the same time, thereby changing the time of flight (TOF) of the pulse-echo. Therefore, the axial stress can be indirectly obtained by measuring the change in TOF of the pulse-echo propagating along the axial direction of the bolt, as shown in Figure 8. Both domestic and foreign researchers have conducted a great deal of research of

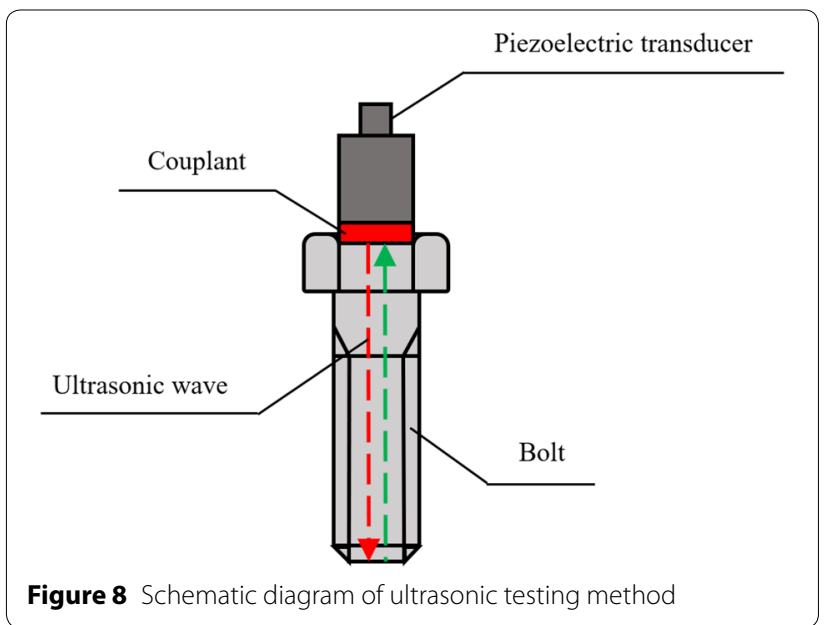

this technology in the theoretical research and equipment development realms, as detailed in Table 2. The researched methods can be roughly classified into monowave method and bi-wave method according to the nature of the measurement.

\subsection{The Mono-wave Method}

The mono-wave method, also called the differential method, refers to the use of a single transverse wave or longitudinal wave to measure the axial stress in a bolt. The longitudinal wave is frequently used in engineering applications because it is more sensitive to changes in stress. An ultrasonic extensometer, which is similar to an ultrasonic thickness gauge, is an early commonly used testing instrument that operates using the mono-wave method. This instrument is designed to measure the change in TOF and report the results as a change in the length of the bolt. Alternatively, it can be used by combining the measured data with constants determined by calibration to provide the axial force in the bolt [13].

The ultrasonic extensometer takes the ultrasonic stretch and the physical stretch of the bolt as intermediate variables, and its principle of detection is shown in Figure 9. First, only the influence of temperature on the ultrasonic velocity and elongation of bolt is considered when using the TOF of the pulse-echo to obtain the ultrasonic stretch. Then, considering the acoustoelastic effect of stress on ultrasonic velocity, the ultrasonic stretch of the bolt is converted into its physical stretch. There are different relationships between the ultrasonic stretch and the physical stretch for different ultrasonic extensometers that can be linear or nonlinear. Finally, the axial force in the bolt is obtained according to the relationship between the axial force $W_{\mathrm{P}}$ and the elongation of the bolt $\Delta L$, also called the physical stretch, as follows: 
Table 2 Classification of major research of the ultrasonic method

\begin{tabular}{lll}
\hline Method & Classification & References \\
\hline $\begin{array}{c}\text { The mono-wave } \\
\text { method }\end{array}$ & Theoretical research & {$[1,15-21,24-27,53]$} \\
$\begin{array}{c}\text { The bi-wave } \\
\text { method }\end{array}$ & Equipment development & {$[11,13,14,22,23,28-33]$} \\
\hline
\end{tabular}

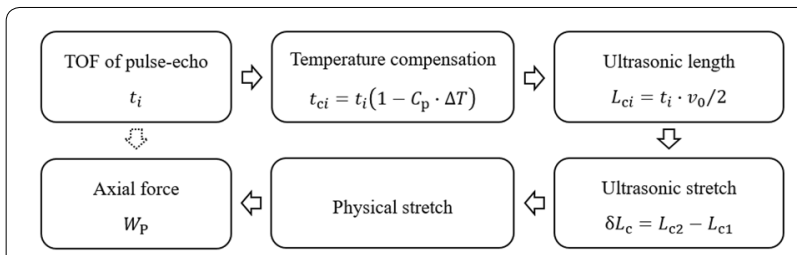

Figure 9 Operating principle of the ultrasonic extensometer

$$
W_{\mathrm{P}}=\frac{A_{\mathrm{e}} \cdot E \cdot \Delta L}{L_{\mathrm{e}}},
$$

where $A_{\mathrm{e}}, E$, and $L_{\mathrm{e}}$ are the area of the effective cross section, the elastic modulus, and the effective stressed length of the unstressed bolt at the reference temperature (a fixed temperature chosen by the manufacturer), respectively.

Because the calculation of axial force must rely upon the measured elongation of bolt plus other calibration data, the measurement of stretch is more accurate than that of axial force, so the ultrasonic extensometer is more commonly used to control the stretch rather than the axial force [13].

Many relevant studies have been conducted abroad using the ultrasonic extensometer. Steblay [14] introduced the pulsed-phase-lock-loop system and the pulseecho system developed by the Denver Research Center of the US Bureau of Mines. The principle of the pulseecho system is similar to that of the ultrasonic extensometer, but bending loading of a bolt cannot be accounted for. Koshti [15] studied the interference fit and bending loading of bolts using the ultrasonic extensometer, further enriching the theoretical basis for the ultrasonic measurement of the axial stress in bolts. For sleeve bolts that provide an interference fit in their cylindrical holes by expanding during assembly, the influence of friction and pressure applied to the shank on the distribution of stress inside the bolt and the corresponding ultrasonic measurements were analyzed [16, 17]. For bolts in a shear joint subjected to bending loads only and for umbilical flange joint bolts experiencing both bending and tensile loads, the phase gradient and physical shift in the received ultrasonic beam and the distortion of the ultrasonic signal caused by the bending geometry and bending stress were analyzed [18-21]. Furthermore, a method for measuring the bending load and deformation of bolts using an ultrasonic extensometer and measures for minimizing the influence of bending on the ultrasonic measurement were proposed. Based on the principles of two commonly used ultrasonic extensometers, the Raymond Boltgage and the StressTel Boltmike, Koshti [22] analyzed potential sources of measurement error, including the fastener characteristics, grip length, transducer coupling, temperature, instrument factors, and the like. Nassar et al. [23] improved the operating principle of an ultrasonic extensometer by continuously updating the velocity of the longitudinal wave, avoiding the need to calibrate the system. They also proposed that the influence of the change in density during the stretching of the bolts was negligible.

The testing principle of an ultrasonic extensometer is relatively complicated as it involves nonlinear relationships and requires the calibration of multiple parameters, neither of which were found to be beneficial to the simplification of the testing process or the improvement of equipment performance in later research. Accordingly, in recent years, researchers have proposed changes in the method of measurement to consider the variation in ultrasonic velocity and the length of bolt simultaneously. This approach avoids the complicated conversion of ultrasonic stretch to physical stretch by directly establishing a theoretical model of the relationship between the measured TOF and axial stress. Without considering the influence of temperature and the effective stressed length, the relationship between the axial stress in a bolt and the measured TOF of the pulse-echo is:

$$
t_{\sigma}=t_{0}\left[1+\left(\frac{1}{E}+A\right) \sigma_{\mathrm{b}}\right]
$$

where $t_{0}$ and $t_{\sigma}$ are the TOFs of the pulse-echo before and after tightening, respectively; $A$ is the acoustoelasticity coefficient; and $\sigma_{\mathrm{b}}$ is the axial stress in the bolt.

A great deal of research has been conducted based on the relationship shown in Eq. (4). Ran et al. [24] provided a brief derivation of the linear relationship between the axial stress and the rate of change in the TOF and proposed the relative acoustic time method, which established the foundation for domestic research of ultrasonic testing technology based on the mono-wave method. The shape factor, which is related to the specifications of the bolt, was proposed to compensate for the effect of the shape of the bolt and the position of the nut on the distribution of stress. In order to improve the accuracy of the TOF measurement, Hirao et al. [25] converted the TOF into a phase shift and resonance frequency shift. The phase shift measurement requires only several 
milliseconds and is suitable for long bolts, while the resonance frequency shift method takes longer and is thus better suited to short bolts. They also observed the complicated effect of the distribution of stress at the nutjoint portion and the deformation in the bolt head on the measurement. Jhang et al. [26] proposed the phase detection technique to measure the TOF of a tone-burst ultrasonic wave. The feasibility of this technique was demonstrated using pure tension and torque application experiments. Jia [11] considered the influence of the effective stressed length and temperature on mono-wave test results and developed a detection system based on field programmable gate array (FPGA) and gigabit ethernet data transmission. This system used the wavelet de-noising method to eliminate interference from high frequency environmental noise, improving the measurement resolution up to $2.131 \mathrm{MPa}$.

In view of the short time and small change in temperature during tightening, Zhu et al. [27-29] determined the difference in the TOF before and after fastening at the same temperature as follows:

$\Delta t=\left(L_{\mathrm{g}}+\delta L\right)\left[\frac{2(1 / E+A)}{v_{0}^{\mathrm{L}}}+\frac{2[A \beta+\alpha(1 / E+A)]}{v_{0}^{\mathrm{L}}}\left(T_{\sigma}-T_{0}\right)\right] \sigma_{\mathrm{b}}$,

where $\Delta t$ is the change in TOF from before to after tightening; $L_{\mathrm{g}}$ is the grip length; $\delta L$ is the stressed length in the bolt head and nut; $v_{0}^{\mathrm{L}}$ is the velocity of the longitudinal wave in the unstressed bolt at the reference temperature; $\beta$ is the thermal expansion coefficient; $\alpha$ is the coefficient describing the influence of temperature on the velocity of longitudinal wave; and $T_{0}$ and $T_{\sigma}$ are the temperatures at the time of calibration and testing, respectively.

The relationship proposed by Zhu et al. is only suitable during the fastening process, and the position of the transducer must remain fixed during the entire assembling procedure to avoid errors caused by inconsistent coupling between the bolt and transducer [27, 28]. With the help of microcomputer technology, they developed a multiple-channel ultrasonic instrument that was used to simultaneously measure the axial stress in 24 bolts. The error of the measured stress was less than $\pm 4 \mathrm{MPa}$ under stresses less than $250 \mathrm{MPa}$ and clamping distances greater than $20 \mathrm{~mm}$ [29]. Based on the former theory, Zhang [30] proposed stress and temperature coefficients related only to the material and corrected for the influence of uncertainty in the clamping length and non-uniform distribution of the stress on the measurement. Sun [1] studied the characteristic curves under the combined action of torsion and tension as well as under solely axial force, and analyzed the influence of the effective clamping length and temperature on the ultrasonic measurement results.
Under the combined influence of axial force and temperature, the relationship between the change in TOF $\Delta t$, the axial stress in the bolt $\sigma_{\mathrm{b}}$, and the change in temperature $\Delta T$ can be expressed as follows [31]:

$$
\Delta t=\frac{2 L_{\mathrm{e}}}{v_{0}}\left(A(T)+\frac{1}{E(T)}\right) \sigma_{\mathrm{b}}+\frac{2 L}{v_{0}}\left(\frac{b(\sigma)}{v_{0}}+\beta(\sigma)\right) \Delta T,
$$

where $v_{0}$ is the ultrasonic velocity in the unstressed bolt at the reference temperature; $A(T)$ is the acoustoelasticity coefficient related to the temperature; $E(T)$ is the elastic modulus related to the temperature; $L$ is the total length of the bolt; $b(\sigma)$ is the stress-dependent coefficient of the ultrasonic TOF as a function of the temperature; and $\beta(\sigma)$ is the thermal expansion coefficient related to the stress.

The relationship between the change in TOF, the axial stress, and the change in temperature is nonlinear and quite complicated. However, the coefficients in Eq. (6) can be ascertained for any given material. Accordingly, Liu [31], Wang [32], and others have directly calibrated these coefficients for different materials with the help of microcomputer technology. This method provides high precision, but is limited to testing under specific working conditions, and as such it is suitable for most, but not all, engineering applications. Zhang [33] applied ultrasonic testing equipment based on this principle shown in Eq. (6) to measure the axial stress in the bolts in a power station and compensated for the uneven distribution of stress caused by the inconsistent cross-sectional area of the bolt.

\subsection{The Bi-wave Method}

The bi-wave method, also called the absolute method, relies on a combination of transverse and longitudinal mode waves to measure the axial force in bolts. At present, several different theoretical models based on the biwave method have been proposed.

Some research directly takes the TOF of the two mode waves as dependent variables. Cunningham et al. [34] took the velocity of the two mode waves in an unstressed bolt, the acoustoelasticity coefficient, and the unstressed length of the bolt as the calibrated parameters and established a theoretical model as follows:

$$
\sigma_{\mathrm{b}}=\frac{t_{\sigma}^{\mathrm{L}} v_{0}^{\mathrm{L}}-t_{\sigma}^{\mathrm{T}} v_{0}^{\mathrm{T}}}{t_{\sigma}^{\mathrm{L}} A_{\mathrm{L}} v_{0}^{\mathrm{L}}-t_{\sigma}^{\mathrm{T}} A_{\mathrm{T}} v_{0}^{\mathrm{T}}-2 l\left(A_{\mathrm{L}}-A_{\mathrm{T}}\right)},
$$

where $t_{\sigma}^{\mathrm{L}}$ and $t_{\sigma}^{\mathrm{T}}$ are the TOFs of longitudinal and transverse waves, respectively, after tightening; $v_{0}^{\mathrm{T}}$ is the velocity of the transverse wave in the unstressed bolt at the reference temperature; $A_{\mathrm{L}}$ and $A_{\mathrm{T}}$ are the 
acoustoelasticity coefficients of longitudinal and transverse waves, respectively; and $l$ is the unstressed length of the bolt. This theoretical model does not consider the effect of temperature on the measurement, and many of the parameters in this method must be separately calibrated, which can have a significant impact on measurement accuracy.

By assuming that the temperature is constant before and after tightening, Jiang et al. $[35,36]$ took the TOF ratio of the two mode waves in the unstressed bolt as the calibrated parameter and considered the influence of the effective stressed length and the change in temperature at the time of calibration and detection. The resulting principle model is as follows:

$$
\sigma_{\mathrm{b}}=\frac{v_{0}^{\mathrm{T}}\left(t_{\sigma}^{\mathrm{L}} x_{0}-t_{\sigma}^{\mathrm{T}}\right)}{2 L_{\mathrm{e}}\left(A_{\mathrm{L}}-A_{\mathrm{T}}\right)},
$$

where $x_{0}$ is the TOF ratio of the two mode waves in the unstressed bolt. Based on this method, an ultrasonic instrument for measuring the stress in a bolt was developed that provided an absolute error of less than $\pm 8 \mathrm{MPa}$ when the stress was less than $250 \mathrm{MPa}$ and the clamping distance was greater than $30 \mathrm{~mm}$ [36].

$\mathrm{Xu}$ et al. conducted a great deal of research of the detection of the axial stress in bolts based on the bi-wave method. They proposed a method for detecting the axial stress when the length of the bolt is unknown, and provided load factor calibration methods for different situations such as low or high load scenarios [37]. In order to avoid errors caused by repeated coupling of the probe, they developed an integrated longitudinal and transverse wave transducer that was capable of exciting the transverse and longitudinal waves either simultaneously or individually [38]. They also constructed an ultrasonic testing system to measure the macroscopic residual stress distribution in the $z$-axis direction [39]. With the help of $L_{C R}$ waves, the influence of the uneven thickness of the measured components and the tensile deformation during the tensile test on the measurement were eliminated. The absolute error of this system was $\pm 20 \mathrm{MPa}$ and the relative error was less than $25 \%$.

The TOF ratio of the two mode waves has been taken as the dependent variable in some research to indirectly obtain the axial stress in bolts. Wu et al. [40] took the TOF ratio of the two mode waves prior to tightening (the reciprocal of the velocity ratio of the two mode waves) as the calibrated parameter to establish the following mathematical model:

$$
\sigma_{\mathrm{b}}=K\left(\frac{t_{0}^{\mathrm{T}}}{t_{0}^{\mathrm{L}}}-\frac{t_{\sigma}^{\mathrm{T}}}{t_{\sigma}^{\mathrm{L}}}\right) /\left(A_{\mathrm{L}} \cdot \frac{t_{\sigma}^{\mathrm{T}}}{t_{\sigma}^{\mathrm{L}}}-A_{\mathrm{T}} \cdot \frac{t_{0}^{\mathrm{T}}}{t_{0}^{\mathrm{L}}}\right),
$$

where $K$ is the shape factor, and $t_{0}^{\mathrm{T}}$ and $t_{0}^{\mathrm{L}}$ are the TOFs of the transverse and longitudinal mode waves, respectively, in the unstressed bolt at the reference temperature. This model established the foundation for domestic research of ultrasonic testing technology based on the bi-wave method.

Based on the theory proposed in Refs. [24] and [40], He et al. [41-43] studied the effect of temperature and shear stress on the ultrasonic measurement of axial stress in a group of bolts tightened in a bridge truss joint. When evaluating the tension in the long-axis connecting bolts of steam turbine generator sets in power plants, Wang et al. [44] summarized the influencing factors on the ultrasonic measurement such as geometric parameters, temperature, and the state of stress in the connected plies and enumerated the difficulties encountered during the research. For common bolts in power stations, Liu et al. [45] developed an ultrasonic instrument with an accuracy of $\pm 10 \mathrm{MPa}$ and provided temperature compensation based on the concept of multi-parameter measurement.

Yasui et al. [46, 47] used the velocity ratio of the two mode waves to derive another theoretical model as follows:

$$
\frac{t_{\sigma}^{\mathrm{T}}}{t_{\sigma}^{\mathrm{L}}}=\frac{v_{0}^{\mathrm{L}}}{v_{0}^{\mathrm{T}}}\left[1+\frac{L_{\mathrm{e}}}{L}\left(A_{\mathrm{L}}-A_{\mathrm{T}}\right) \sigma_{\mathrm{b}}\right],
$$

Evaluating the short and highly stressed bolts used in automobile assembly, they accounted for the influence of nonlinear extension caused by the local plastic deformation of the bolt as well as the non-uniform distribution of stress in the cross section. Doing so, they developed a testing system that could estimate the axial load in bolts with an error as small as 15\% when the tensile spring constant was greater than $900 \mathrm{kN} / \mathrm{mm}$ [47]. On the basis of the theoretical model in Eq. (10), Chaki et al. [48] proposed a method for the systematic calibration of parameters such as the effective stressed length that does not require a large number of calibration tests. They also pointed out the influence of acoustic diffraction on axial tension detection. This method provided an accuracy within $\pm 10 \%$ and a $95 \%$ reliability.

The mode-converted ultrasound method is a new method used to measure the axial stress in bolts that avoids the error caused by the repeated coupling of the transducer. Kim et al. [6] used such oblique-incident ultrasound to simultaneously generate transverse and longitudinal waves in the subject bolt. The principle of detection is shown in Figure 10: mode conversion occurs when the ultrasonic wave is obliquely incident at a small angle relative to the end of the bolt. The longitudinal wave $(\mathrm{L})$ and transverse wave $(\mathrm{T})$ in Figure 10 is generated by the refraction of the incident wave. When the two 


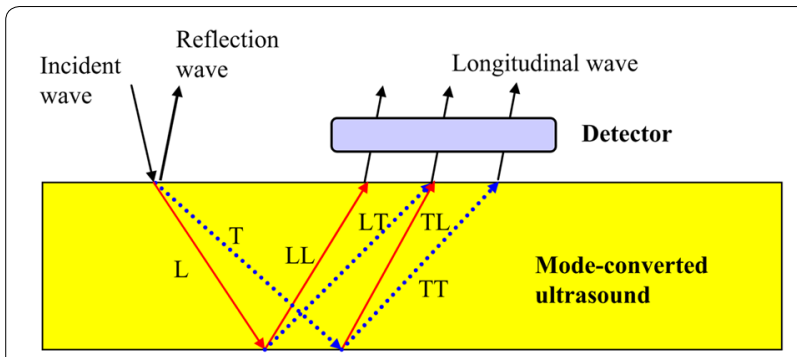

Figure 10 Ultrasonic mode conversion in a bounded solid [6]

mode waves hit the bottom surface of the bolt, secondary mode conversion occurs as a result of this reflection, providing the LL, LT, TL, and TT mode waves shown in Figure 10. The principle models of the relationship between the TOF and the axial stress were then established by analyzing the propagation paths of the LL and LT mode waves in a short bolt and long bolt, respectively. The signal received using the mode-converted ultrasound method is shown in Figure 11. It has been observed that this method provides sufficient resolution to estimate an axial stress less than $10 \%$ of the yield stress of the material with a maximum error of $5 \%$.

Ding et al. $[49,50]$ used the electromagnetic acoustic transducer (EMAT) to generate a transverse wave at the end of the bolt and obtained the longitudinal wave from the reflection of the transverse wave using mode conversion, as shown in Figure 12. In this figure, the bolt is simplified as a cylindrical specimen, $S_{1}$ is the path of the incident transverse wave, $S_{2}$ is the path of the reflected transverse wave, $S_{3}$ is the path of the reflected longitudinal wave, $\alpha_{\mathrm{T}}$ is the emission angle of the incident transverse wave, $\theta_{\mathrm{T}}$ is the incident angle of the incident transverse wave, $\gamma_{\mathrm{T}}$ is the reflection angle of the reflected transverse wave, and $\gamma_{\mathrm{L}}$ is the reflection angle of the reflected longitudinal wave. Through the path analysis of

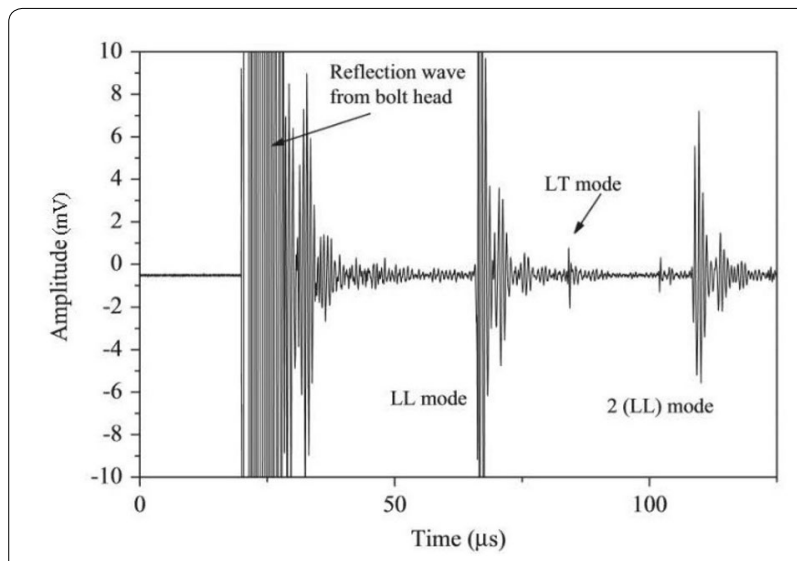

Figure 11 Mode-converted ultrasound from bolt specimen [6]

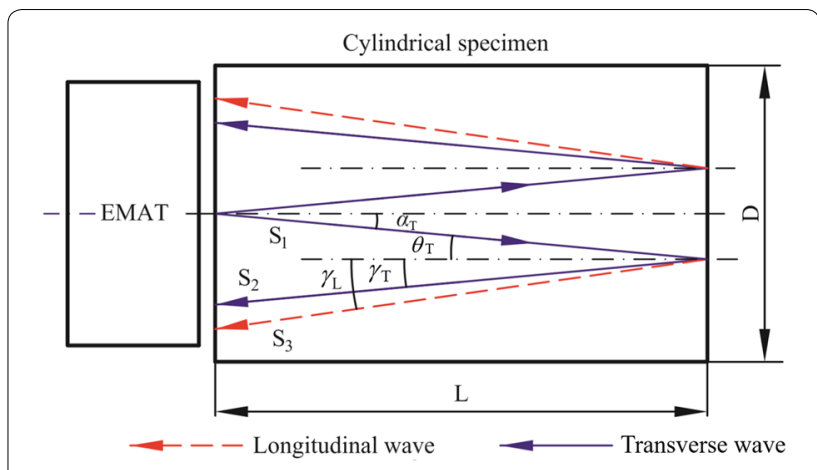

Figure 12 Ray paths of a mode-converted ultrasound of a cylindrical specimen [49]

the mode-converted ultrasound, the following theoretical model was established [49]:

$$
\frac{t_{1-3}}{t_{1-2}}=\frac{1}{2}+\frac{v_{0}^{\mathrm{T}}}{2 v_{0}^{\mathrm{L}}}+\frac{v_{0}^{\mathrm{T}}}{2 v_{0}^{\mathrm{L}}} \cdot\left(A_{\mathrm{T}}-A_{\mathrm{L}}\right) \sigma_{\mathrm{b}},
$$

where $t_{1-2}$ and $t_{1-3}$ are the TOFs of the TL mode wave and TT mode wave, respectively.

On this basis, an electromagnetic ultrasonic system for measuring the axial force in in-service bolts was developed for use with M24-M30 bolts. The maximum length of the bolts was $200 \mathrm{~mm}$ and the relative measurement error was less than $10 \%$ [50].

Similar to the testing principle in Refs. [31] and [32], Zhu et al. [51] collected a large quantity of data with the help of numerical computations and calculated 16 coefficients for a three-order equation with two variables to establish a theoretical model. The ultrasonic high-temperature bolt stress monitor developed based on this principle can be used at temperatures between $0{ }^{\circ} \mathrm{C}$ and $350{ }^{\circ} \mathrm{C}$ for more than two years, and the measurement accuracy was better than $\pm 9.8 \mathrm{MPa}$. Liu [52] developed an ultrasonic axial stress meter with a single-chip microcomputer at its core that provided an accuracy of $\pm 1 \mathrm{MPa}$. In actual measurements using this meter, only the TOF of the two mode waves needs to be measured, the temperature does not. This method is particularly useful in engineering applications with a narrow range of detection.

At present, research of the mono-wave and bi-wave methods is only applicable to bolts that exhibit elastic deformation. The mono-wave method requires fewer measurements, is easy to operate, and provides high accuracy, and accordingly equipment using this method has been put into service in many engineering applications. However, under the mono-wave method it is necessary to measure the TOF of the pulse-echo propagating along the unstressed bolt, and accordingly this method 
is mainly suitable for the control and verification of the preload applied during the assembly of the bolt. When using the bi-wave method, although the transverse wave is less sensitive to the change in stress than the longitudinal wave, resulting in lower accuracy compared to the mono-wave method, the length of bolt is not required in advance, thus this method provides easier detection of the axial force in an already tightened bolt.

\section{Analysis of Influencing Factors}

\subsection{Effective Stressed Length}

The axial stress in a bolt after tightening is not uniformly distributed; only part of the bolt-the effective stressed length-is subjected to stress. The effective stressed length of the bolt $L_{\mathrm{e}}$ includes the clamping portion of the bolt shank $L_{\mathrm{g}}$ and part of the bolt head and nut, as shown in Figure 13. Some relevant research has been constructed on the determination of the effective stressed length. Considering the influence of the shape of the bolt and the position of the nut, Ran et al. [24] proposed a theoretical formula and experimental method for determining the shape factor of a bolt or screw. However, the proposed process is complex and not suitable for theoretical research and engineering applications. Because the effective stressed length of a bolt is not easy to accurately obtain, empirical formulas are typically used in engineering applications to simplify the process of calculation and measurement. It was proposed that the effective stressed length of a bolt can be expressed as the sum of the actual clamping distance between the nut and head of the bolt, one-half of the equivalent diameter of the threaded portion of the shank, and one-third of the diameter of the unthreaded bolt shank [53]. The effective stressed length has also been proposed as the sum of the thickness of the connected plies and the equivalent diameter of the threaded shank [1]. However, these empirical formulas are all approximate calculations and their results differ from the actual effective stressed lengths of different bolts, introducing various errors into the measurements. Accordingly, in order to determine the relevant law describing effective stressed length, Chaki [48] conducted comparative tests of bolts of the same diameter,

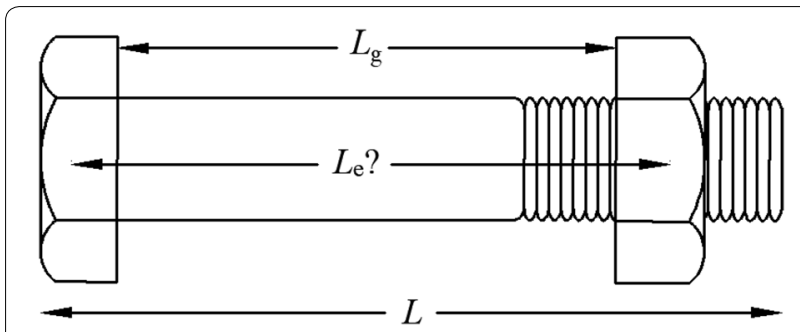

Figure 13 Model of axially loaded bolt [48] obtaining the following relationship for different total lengths $L$ and different grip distances $L_{\mathrm{g}}$ :

$$
L_{\mathrm{e}}^{j}-L_{\mathrm{g}}^{j}=L_{\mathrm{e}}^{k}-L_{\mathrm{g}}^{k}=\delta L,
$$

where $L_{\mathrm{e}}^{j}$ and $L_{\mathrm{e}}^{k}$ are different effective stressed lengths; $L_{\mathrm{g}}^{j}$ and $L_{\mathrm{g}}^{k}$ are different grip distances; and $\delta L$ is the correction factor, that is, the stressed length of the partial area in the bolt head and nut.

Chaki pointed out that $\delta L$ depends only on the bolt geometry (diameter, thickness of the bolt head and nut, thread), regardless of the total length of the bolt or actual grip distance [48]. Figure 14 shows a schematic of mechanical bolt assembly used in many industries that have the same bolt material and diameter, but different lengths and different tightening configurations. The bolts in these assemblies all have the same $\delta L$ and thus the testing equipment would need to be calibrated only once for such bolts, avoiding cumbersome theoretical calculations and a large number of calibrations.

Currently, ultrasonic testing to determine the axial stress in bolts is conducted based on the assumption that the stress is uniformly distributed throughout the effective stressed region, meaning that the reported value is the average stress. However, the change in stress across the continuum of the bolt is unlikely to be abrupt; there must be a slow change of stress at the edge of the effective stressed region. To address this problem, Pan et al. [54] obtained the distribution of stress in the axial direction of a bolt using a finite element simulation, as shown in Figure 15. They also proposed the shape factor concept and calculation method based on the differential method and established a mathematical model describing the relationship between the shape factor and the clamping distance, further improving ultrasonic testing accuracy. In future research, it will be necessary to convert the measured average value into the maximum axial stress in the most dangerous section to check the safety factor of the bolt.

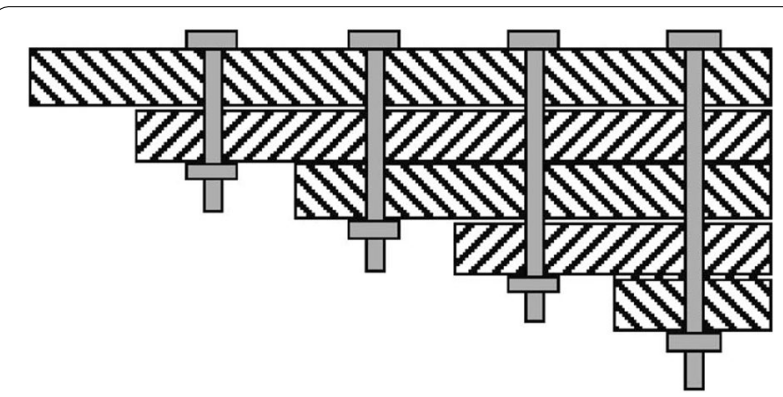

Figure 14 Example of a mechanical bolt assembly [48] 


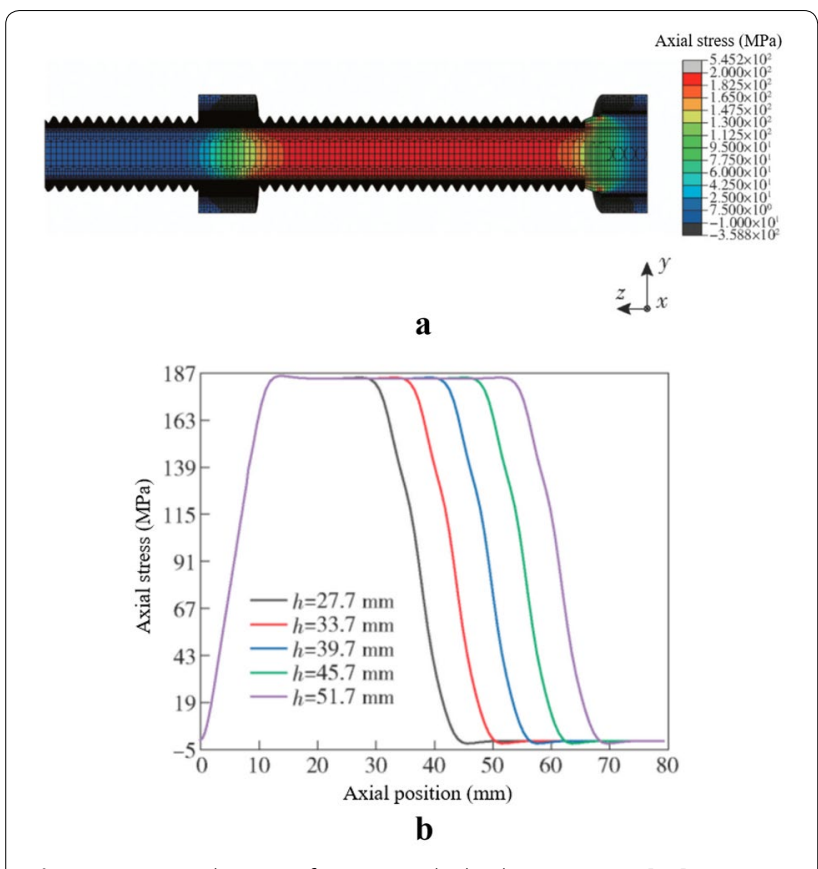

Figure 15 Distribution of stress in a bolted connection [54]:

\subsection{Temperature of the Bolt}

The effect of temperature on the ultrasonic measurement is manifested in two ways. On the one hand, the change in temperature causes the length of the bolt to change; that is, the thermal expansion effect. Generally, the thermal expansion coefficient of steel is on the order of $10^{-5}$, and the resulting error is accordingly small. On the other hand, the change in temperature affects the elastic modulus and density of the material, which causes the ultrasonic velocity to change. The influence of temperature on the ultrasonic velocity is on the order of $10^{-4} /{ }^{\circ} \mathrm{C}$ which is of the same order of magnitude as the effect of axial stress, so the resulting error can be relatively large [55]. Therefore, temperature must be monitored and compensated for in real time during the detection of the axial stress in bolts.

Under the condition that the axial stress $\sigma_{\mathrm{b}}$ and the change in temperature $\Delta T$ exist simultaneously, the relationship between the TOF of the pulse-echo, the temperature, and the stress is as follows [41]:

$$
t\left(T_{\sigma}, \sigma_{\mathrm{b}}\right)=t\left(T_{0}, 0\right)\left[1+(\beta-\alpha) \Delta T+K(T) \sigma_{\mathrm{b}}\right],
$$

where $t\left(T_{\sigma}, \sigma_{\mathrm{b}}\right)$ is the TOF when the temperature is $T_{\sigma}$ and the stress is $\sigma_{\mathrm{b}} ; t\left(T_{0}, 0\right)$ is the TOF when the temperature is $T_{0}$ and the stress is 0 ; and $K(T)$ is the coefficient related to the temperature.

It can be seen from Eq. (13) that the temperature not only affects the slope of the resulting curve but also the offset of its zero point. He et al. [41] determined that when the change in temperature is small, it mainly affects the zero point of the curve while its effect on the slope can be neglected in the actual measurement. This viewpoint was confirmed by measuring the relationship between the difference in the TOF of the longitudinal wave, stress, and temperature for M30 carbon steel bolts, as shown in Figure 16(a). When the change in temperature is large, the relationships between the differences in the TOF of the two mode waves and the stress in M14 $35 \mathrm{CrMo}$ bolts at $30{ }^{\circ} \mathrm{C}$ and $360{ }^{\circ} \mathrm{C}$ are measured with the results shown in Figure 16(b), in which it can be seen that this relationship is not simply a translation when the temperature changes significantly [35]. At the same time, it
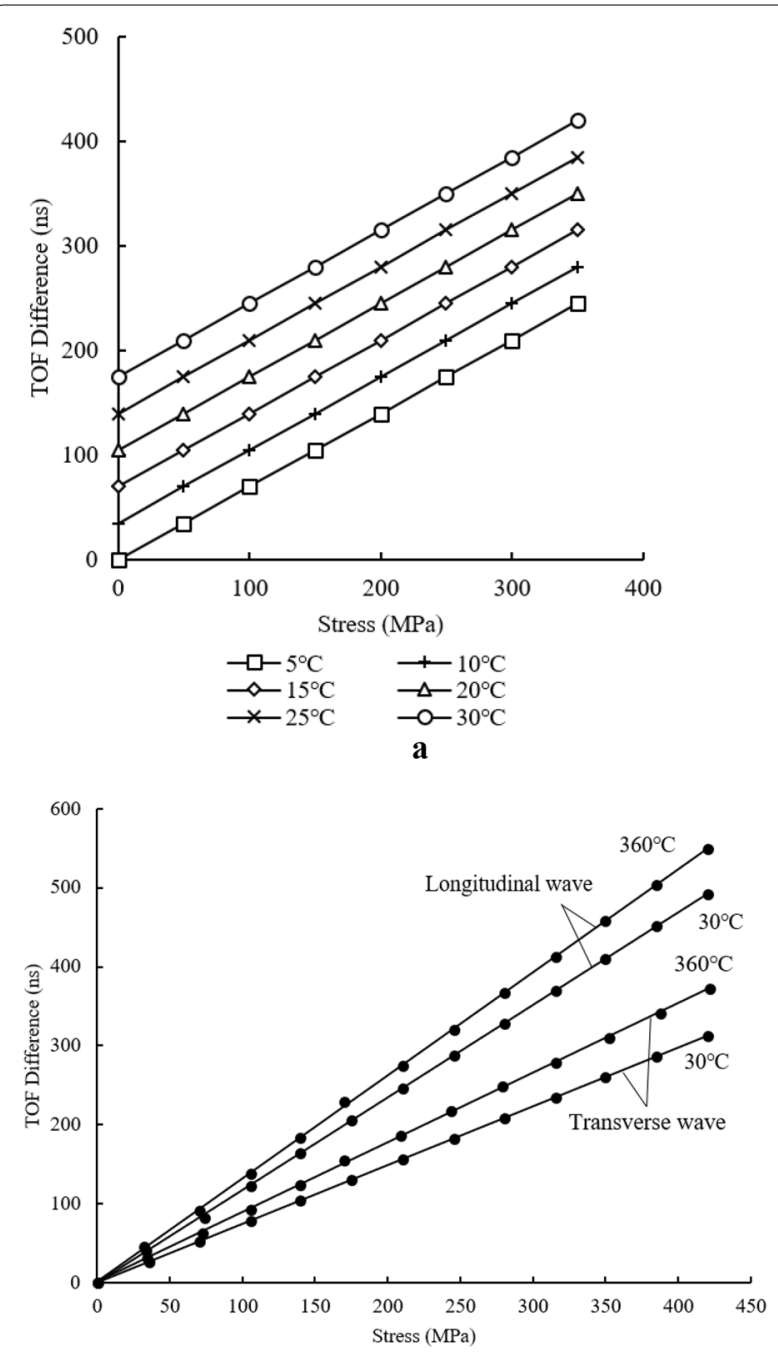

b

Figure 16 Relationship between the TOF difference and stress $[35,56]$ : a small change in temperature, and $\mathbf{b}$ significant change in temperature 
has been proposed that the TOF of the transverse wave is about twice that of the longitudinal wave, and that the slope of the transverse wave curve is about two-thirds of that for the longitudinal wave at the same temperature [45].

If the change in temperature is too large, the effect of temperature on the slope of the TOF difference-stress curve should be considered in the temperature compensation. In this case, however, the necessary calibration is typically complicated and many parameters require calibration, resulting in poor measurement accuracy. Considering that most measurements are normally carried out under room temperature and that the change in temperature is not significant, the relationship between the temperature and TOF in an unstressed bolt can be used as compensation for the actual measurement.

\section{Discussion of Development Prospects}

\subsection{Ultrasonic Coupling Technology}

Although a great deal of research has been conducted on ultrasonic theory and equipment to determine the axial stress in bolts, as the most basic technical parameter, the coupling between the ultrasonic transducer and the surface of bolt remains the primary problem to be solved. Currently, piezoelectric transducers are widely used. A poor bolt surface condition and unstable coupling properties will inevitably affect the accuracy of ultrasonic detection [25]. In order to ensure good coupling performance, it is necessary to process the end face of the subject bolts using methods such as turning and polishing prior to measurement, but this consumes a great deal of time and labor. Additionally, the applied preload is only $75 \%$ of the design load for most bolts, and the ultrasonic TOF is measured at the nanosecond level. During measurement, the ultrasonic wave passes through the coupling layer twice, thus unevenness and inconsistency of the coupling layer can produce errors on the same order of magnitude as the measured value [1]. Therefore, for each measurement, the transducer should be installed in the same position and the pressure on the end face of the bolt should be equal. These are very demanding requirements to meet in actual field measurement. In order to improve coupling performance, the use of EMAT and the permanent mounted transducer system (PMTS) have gradually attracted the attention of researchers in recent years.

As an emerging technology in the field of nondestructive testing, EMAT has been increasingly applied to ultrasonic testing due to its advantages, which include no coupling or high-quality workpiece surface condition requirements, a simple device, and fast

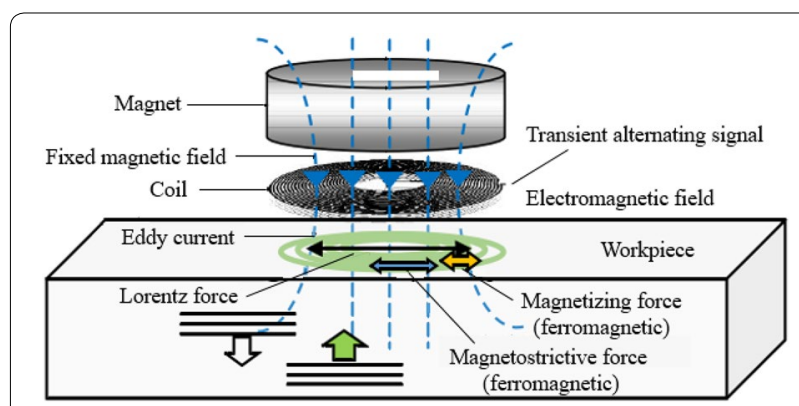

Figure 17 Working mechanism of EMAT $[60,61]$

measurement speed [57-59]. The EMAT consists of a high-frequency induction coil, an external bias magnetic field, and the workpiece to be tested. Its working mechanism involves the Lorentz force, magnetostrictive force, and magnetizing force, as shown in Figure 17. The workpiece is used as a part of the EMAT and so different workpiece materials will determine different mechanisms of ultrasonic excitation.

With the improving performance of piezoelectric ceramics and the emergence of diverse preparation methods, the use of PMTS technology has increased. This technique bonds a piezoelectric chip to the head of the subject bolt or directly deposits the piezoelectric material onto the end face of the bolt by means of ion vapor deposition (IVD) or pulsed laser deposition (PLD) to form a composite sensor [1, 62, 63]. The bolt then becomes a piezoelectric sensing bolt, as shown in Figure 18. During measurement, an ultrasonic exciter must be in contact with the sensor and an electric pulse is applied to the piezoelectric ceramic to generate ultrasonic waves in the bolt, avoiding the need to apply a couplant. This technology has been applied in advanced

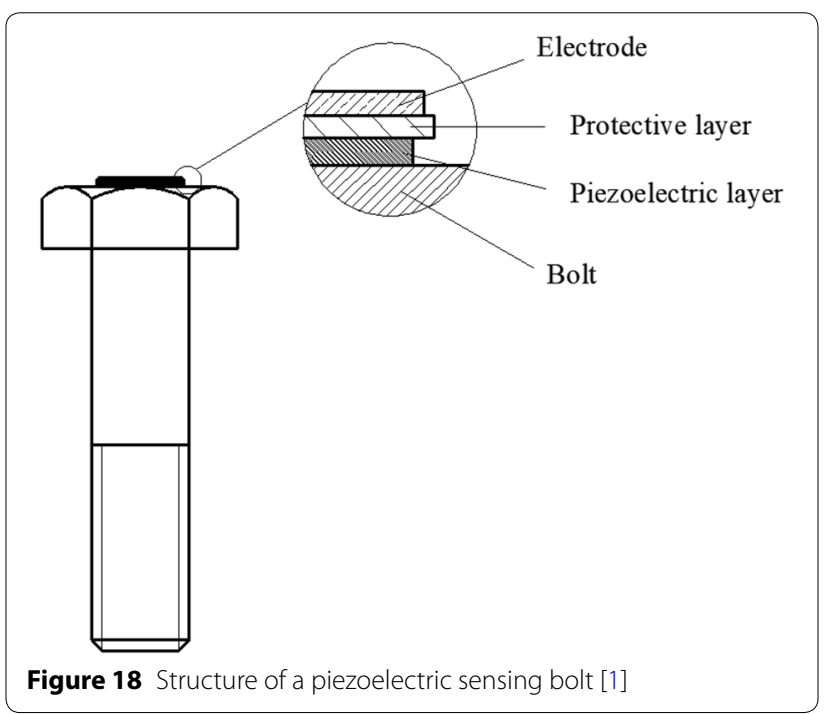


industrial fields such as aerospace and automobiles manufacturing.

At present, EMAT and PMTS are gradually replacing the piezoelectric transducer in the development of ultrasonic measurement technology. However, both technologies are immature and there remain many difficulties to overcome before widespread application is practical or appropriate. For EMAT, the process of energy transduction involves the multi-physics coupling of electro-magnetic-force-acoustic, which results in low conversion efficiency and difficulty in exciting the longitudinal wave on the end faces of ferromagnetic bolts $[64,65]$. Accordingly, a shear-wave EMAT has been used for detection but energy compensation was required [25, 49]. Currently, the primary difficulty of applying EMAT is the need to optimize its excitation and receiving devices to improve the energy conversion efficiency. The application of PMTS requires special handling of the bolt, which makes it difficult to apply them to ordinary industrial production methods. Moreover, the process of preparing the film on the end of the bolt is complicated and when disposed, the processed bolt is harmful to the environment due to lead content. Research of lead-free piezoelectric and ferroelectric materials has accordingly become a hot spot in the field of PMTS research [63].

\subsection{Residual Tension Testing of Bolts Assembled to Yield}

In service, the axial stress in bolts will gradually decrease due to the influence of the working load and external environment. So, the residual axial force in a bolt is an important indicator of the effectiveness of the bolted connection design $[66,67]$. The detection of residual axial force in bolts with elastic deformation can be achieved by the methods discussed in Section 4. However, whether tightened by the yield point method or subjected to external loads, the bolt will inevitably reach its yield strength during service, causing plastic deformation. Most of the ultrasonic methods proposed at present are not applicable to bolts exhibiting plastic deformation, and there are few studies of methods for detecting the residual axial force in bolts that have been assembled to yield.

There is a pseudo-axial force caused by plastic deformation in a yielding bolt. Accurate identification and measurement of this pseudo-axial force is the key to measure the residual tension in the yielding bolt [68]. According to whether the post-yield behavior is included, the property curve based on the axial stress and the change in TOF can be classified into a linear curve and a nonlinear curve, as shown in Figure 19. In this figure, the bolt is tightened along the path FGA to the yield strength, then

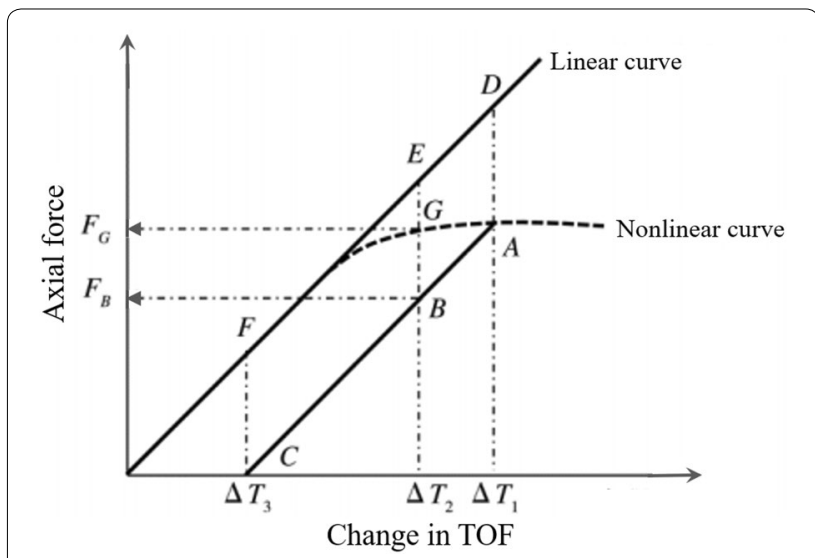

Figure 19 Property curve of bolts [68]

loosened along the path $\mathrm{ABC}$. The path $\mathrm{ABC}$ is generally considered to have the same slope as the linear segment FED. The axial force CF corresponding to the change in TOF after loosening can then be identified as the magnitude of the pseudo-axial force.

There are two methods for measuring the residual tension in bolts that have been assembled to yield: the disassembling method and property curve method, both of which essentially measure the pseudo-axial force in the bolt. The disassembling method relies only upon the linear property curve, but the bolt must be completely disassembled during the test. Accordingly, this method is mainly used in laboratory research and is not suitable for the monitoring of residual axial force during field service. The property curve method does not require disassembly of the bolt, but does require that both the linear and nonlinear curves be obtained. And it is necessary to continuously measure the axial stress in the bolt in real time during assembly to accurately obtain the initial position of axial force attenuation [68]. Both methods are based on the static tensile curve of metal. However, nonparallelism between the loading path and the unloading path has been experimentally confirmed [69]. In order to reduce the accompanying measuring error, it has been proposed that the loosening curve be calibrated to decrease the measurement deviation to within $\pm 2 \%$ when the attenuation of the axial force is less than $30 \%$ [69].

Bolted connections are widely used in various equipment and structures. In particular, bolts tightened to yield are often used to provide more precise control of the clamping force. Therefore, the measurement of residual axial force is of great significance for continued safety monitoring. At present, the two proposed methods for measuring the residual axial force in yielded bolts each have their own limitations. To provide appropriate and 
accurate detection of the axial stress in yielded bolts, further research is required to enrich the theory of axial stress measurement in yielded bolts.

\section{Conclusions and Outlook}

In this paper, the stress and deformation states of bolts under different conditions are briefly discussed, conventional measurement methods are summarized and compared, and the current state of ultrasonic testing technology for measuring the axial stress in bolts is reviewed and analyzed. The following conclusions are drawn:

1. Conventional measurement methods provide indirect measurements, requiring extensive conversion processes that can be adversely affected by many factors. It is difficult for most of these methods to accommodate the requirements of rapid and highprecision assembly. Moreover, these methods are only applicable to the control of preload and can be quite inconvenient when measuring the axial stress in a tightened bolt.

2. Ultrasonic axial stress measurement methods can be classified into mono-wave method and bi-wave method. The mono-wave method must first measure the ultrasonic TOF of the unstressed bolt and is suitable for the control of preload in an assembly. The biwave method does not require that the length of the bolt be known in advance and is applicable for the detection of the axial stress in tightened bolts. Both methods are suitable for the detection of axial stress in bolts under elastic deformation but have certain limitations for bolts that have yielded.

3. The effective stressed length and temperature are the two main factors influencing the accuracy of axial stress measurements in bolts. The effective stressed length is only related to the geometry of the bolt (diameter, thickness of bolt head and nut, thread) and can be determined by calibration of bolts of the same specification. Different temperature variation ranges have different effects on the measurement, but only the change in the zero point of the TOF differencestress curve is typically considered.

4. Newer ultrasonic coupling technologies, EMAT and PMTS, are gradually replacing traditional piezoelectric transducers due to their unique advantages. However, the efficiency of EMAT is low, while the operation of PMAT is troublesome, and the treated bolts are difficult to dispose of due to environmental hazards of the applied transducer. Clearly, there still are many issues to be addressed before these methods can be widely applied.
5. With the gradual popularization of the yield point bolt installation method, the determination of the residual axial stress in bolts that have been assembled to yield has attracted much attention from researchers. However, each of the current methods for determining residual axial load, the disassembling method and the property curve method, have their own disadvantages. There is still no mature method for measuring the residual axial force in a yielded bolt. More research and analysis need to be conducted before accurate determination of axial stress in yielded bolts can be ensured.

\section{Acknowledgements}

We would like to thank Editage [www.editage.cn] for English language editing.

\section{Authors' Contributions}

QP put forward the framework and content of the paper; RP wrote the manuscript; CS shared many fundamental ideas in the ultrasonic measurement technology. MC and XX assisted in modifying the structure and content of this paper. All authors read and approved the final manuscript.

\section{Authors' Information}

Qinxue Pan, born in 1981, is currently an associate professor at Beijing Institute of Technology, China. He received his PhD degree from Kagawa University, Japan, in 2010. His research interests include ultrasonic nondestructive testing technology, residual stress detection and characterization. Tel: +86-13716096968.

Ruipeng Pan, born in 1995, is currently a master candidate at School of Mechanical Engineering, Beijing Institute of Technology, China. His research interests include ultrasonic measurement technology for the axial stress in high-strength bolts.

Chang Shao, born in 1990, is currently a master candidate at School of Mechanical Engineering, Beijing Institute of Technology, China. His research interests include ultrasonic measurement technology for the axial stress in bolts.

Meile Chang, born in 1996, is currently a master candidate at School of Mechanical Engineering, Beijing Institute of Technology, China. Her research interests include ultrasonic nonlinear measurement for the axial stress in bolts.

Xiaoyu Xu, born in 1996, is currently a master candidate at School of Mechanical Engineering, Beijing Institute of Technology, China. His research interests include ultrasonic testing technology for residual stress in polymer bonded explosives.

\section{Funding}

Supported by Project of Basic Technology Research which is funded by Technology and Quality Division of the Ministry of Industry and Information Technology (Grant No. JSZL2017602B002).

\section{Competing Interests}

The authors declare that they have no competing interests.

\section{Author Details}

${ }^{1}$ School of Mechanical Engineering, Beijing Institute of Technology, Beijing 100081, China. ${ }^{2}$ Key Laboratory of Fundamental Science for Advanced Machining, Beijing Institute of Technology, Beijing 100081, China.

Received: 16 April 2019 Revised: 12 December 2019 Accepted: 9 January 2020

Published online: 11 February 2020 


\section{References}

[1] G F Sun. Research on axial force of bolt tightening based on the ultrasonic measurement technology. Hangzhou: Zhejiang University of Technology, 2012. (in Chinese)

[2] Z D Zhu, H Lin. Comparison and application research of torque method and torque/rotation method based on bolt assembly technology. Design and Manufacture of Diesel Engine, 2005, 14(2): 40-43. (in Chinese)

[3] J J Li. Control of bolt preload during assembly. Materials and Processes, 2010, 41: 57-59. (in Chinese)

[4] J H Wang. Mechanical design foundation. Wuhan: Huazhong University of Science and Technology Press, 2008: 179-182. (in Chinese)

[5] K Zhou, M H Liu. Analysis of calculation and control methods of the bolt preload in the flange bolts connection. New Technology and New Process, 2010(8): 26-28. (in Chinese)

[6] N Kim, M Hong. Measurement of axial stress using mode-converted ultrasound. NDT and E International, 2009, 42(3): 164-169.

[7] W Huang, J Q Chen. Reliability analysis of tightening bolts with moment method and rotation angle method. Equipment Manufacturing Technology, 2006(4): 6-8. (in Chinese)

[8] G ZWu. Calculation of tightening stress and torque of bolt. Internal Combustion Engine and Powerplant, 2012(1): 39-41. (in Chinese)

[9] C Li, Y J Zhu, Z X Hou. Theoretical and testing study on tightening behavior of high-strength bolts and turn-of-the-nut method. Building Structure, 2013, 43(S1): 448-452. (in Chinese)

[10] J S Zheng. Studies on techniques and experiments of the bolts tightening for engine's cylinder head. Shanghai: Shanghai Jiao Tong University, 2008. (in (hinese)

[11] X Jia. Research on bolt axial stress detection system based on acoustoelastic effect. Chengdu: Southwest Jiaotong University, 2018. (in Chinese)

[12] S Liu. Ultrasonic nondestructive testing technology of the distribution of residual stress for tiny component. Beijing: Beijing Institute of Technology, 2016. (in Chinese)

[13] J H Bickford. An introduction to the design and behavior of bolted joints. New York: Marcel Dekker, 1990: 299-347.

[14] B J Steblay. New instrumentation for roof bolt load measurement. IEEE Transactions on Industry Applications, 1987, 23(4): 731-735.

[15] A M Koshti. Ultrasonic measurement of loads in bolts used in structural joints. NESC NDE Face to Face Meeting, Michoud, LA, United States, March 24, 2015.

[16] A M Koshti. Preload measurement in sleeve bolts using an ultrasonic technique. Materials Evaluation, 1996, 54(2): 308-313.

[17] A M Koshti. Simulation of ultrasonic preload measurement on a bolt in an interference fit joint. NDE For Health Monitoring and Diagnostics, San Diego, California, United States, June 11, 2002: 423-437.

[18] A M Koshti. Ultrasonic measurement of the bending of a bolt in a shear joint. Experimental Mechanics, 1998, 38(4): 270-277.

[19] A M Koshti. Simulation of ultrasonic measurement on a bolt in a shear joint. NDE For Health Monitoring and Diagnostics, San Diego, California, United States, June 11, 2002: 411-422.

[20] A M Koshti. Effect of bending on ultrasonic preload measurements in bolts. 6th Annual International Symposium on NDE for Health Monitoring and Diagnostics, Newport Beach, CA, United States, July 24, 2001: 143-154.

[21] A M Koshti. Simulation of effect of bending stress on the ultrasonic beam. NDE For Health Monitoring and Diagnostics, San Diego, California, United States, June 11, 2002: 148-156.

[22] A M Koshti. Estimation of accuracy in ultrasonic preload measurements. 6th Annual International Symposium on NDE for Health Monitoring and Diagnostics, Newport Beach, CA, United States, July 24, 2001: 300-311.

[23] S Nassar, G Grzadzinski. Ultrasonic control of bolt tightening: U.S. Patent 7, 360, 435. 2006-6-29

[24] Q F Ran, X R Fei, C D Deng, et al. Application of ultrasonic technique to bolt stress measurement. Acta Mechanica Solida Sinica, 1982(1): 64-70. (in (hinese)

[25] M Hirao, H Ogi, H Yasui. Contactless measurement of bolt axial stress using a shear-wave electromagnetic acoustic transducer. NDT and E International, 2001, 34(3): 179-183.

[26] KY Jhang, H H Quan, J Ha, et al. Estimation of clamping force in hightension bolts through ultrasonic velocity measurement. Ultrasonics, 2006, 44(8): 1339-1342.
[27] S M Zhu, ZT Jiang, J Lu, et al. Ultrasonic transit time difference method for measuring the prestress force of the bolt. Technical Acoustics, 1995, 14(4): 181-186. (in Chinese)

[28] ZT Jiang, S M Zhu, J Lu, et al. The principle and error analyses of microcomputer-based ultrasonic instrument for the measurement of axial bolt stress. Applied Acoustics, 1996, 15(4): 1-5. (in Chinese)

[29] ZT Jiang, S M Zhu. Microcomputer-based multiple channels ultrasonic instrument for measuring stress of bolt. Chinese Journal of Scientific Instrument, 2000, 21(4): 367-370. (in Chinese)

[30] J Zhang. Research on the ultrasonic measurement technology of bolt tension based on the sonic-elasticity principle. Hangzhou: Zhejiang University, 2005. (in Chinese)

[31] Z Q Liu, J W Liu, C L Li, et al. Ultrasonic measurement method of bolt fastening force. Valve, 1996(2): 29-31. (in Chinese)

[32] L Wang. Ultrasonic technology and temperature correction for bolt stress measurement. Automobile Technology and Material, 1999(6): 37-38, 41. (in Chinese)

[33] D G Zhang. Ultrasonic stress measurement of bolts in a power station. Hebei Electric Power, 2000, 19(4): 27-29. (in Chinese)

[34] B Cunningham, A C Holt, G C Johnson. Sensitivity of an ultrasonic technique for axial stress determination. Review of Progress in Quantitative Nondestructive Evaluation, 1988: 1405-1412.

[35] ZT Jiang. Effect of temperature on ultrasonic velocity and stress measurement. NDT, 1999, 21(6): 245-248. (in Chinese)

[36] ZT Jiang, S Q Zhang, J C Hu, et al. A new type of microcomputer-based ultrasonic longitudinal waves and transverse wave instrument for measurement of stress in bolt. Acta Mechanica Solida Sinica, 2001, 22(4): 415-420. (in Chinese)

[37] C G Xu, X Li, Q X Pan, et al. Bolt stress measurements by ultrasonic nondestructive methods. Journal of Applied Acoustics, 2014, 33(2): 102-106. (in Chinese)

[38] C G Xu, X Li, Q X Pan, et al. A shear and longitudinal wave ultrasonic sensor for axial stress detection of bolts: Chinese Patent 102865954A 2013-01-09. (in Chinese)

[39] C G Xu, H X Li, J F Wang, et al. Ultrasonic shear and longitudinal wave testing method of residual stress. Acta Acustica, 2017, 42(2): 195-204. (in Chinese)

[40] K CWu, S S Lin. Improvement of an ultrasonic method for measuring stress in bolt. Journal of Huazhong University of Science and Technology (Natural Science Edition), 1988, 16(1): 171-176. (in Chinese)

[41] C F He, K C Wu. Influence of temperature on ultrasonic measurement of axial stress in high strength bolts. Journal of Experimental Mechanics, 1992 7(3): 238-244. (in Chinese)

[42] C F He, K C Wu. Influence of shear stress on the ultrasonic measurement of the axial stress of high strength bolts. Acta Mechanica Solida Sinica, 1992, 13(4): 337-342. (in Chinese)

[43] C F He, K C Wu, J Z Shi, et al. The measurement of bridge bolt-stress by using ultrasonic technique. Journal of Experimental Mechanics, 1994, 9(1): 74-78. (in Chinese)

[44] Z Q Wang, L D Liang, W Huang, et al. Review of ultrasonic method for measuring bolt stress. Information on Electric Power, 1994, (3): 21-25. (in Chinese)

[45] Z Q Liu, L Wang. An axial bolt stress meter with the ultrasonic time and temperature as parameters. Chinese Journal of Scientific Instrument, 1996, 17(6): 662-665. (in Chinese)

[46] H Yasui, H Tanaka, I Fujii, et al. Ultrasonic measurement of axial stress in short bolts with consideration of nonlinear deformation. JSME International Journal, 2008, 42(1): 111-118.

[47] H Yasui, K Kawashima. Acoustoelastic measurement of bolt axial load with velocity ratio method. 15th World Conference on Non-Destructive Testing, Rome, October 15-21, 2000: 750-756.

[48] S Chaki, G Corneloup, I Lillamand, et al. Combination of longitudinal and transverse ultrasonic waves for in situ control of the tightening of bolts. Transactions of the ASME: Journal of Pressure Vessel Technology, 2007 129(3): 383-390.

[49] X Ding, X J Wu, Y G Wang. Bolt axial stress measurement based on a mode-converted ultrasound method using an electromagnetic acoustic transducer. Ultrasonics, 2014, 54(3): 914-920.

[50] X Ding, X J Wu. Development of EMAT based axial load measurement system for in-service bolts. Nondestructive Testing, 2016, 38(6): 48-52, 64. (in Chinese) 
[51] S M Zhu, M Z Xiao, J Lu, et al. Development of the ultrasonic high temperature bolt stress monitor. Journal of Tongji University (Natural Science), 1991, 19(4): 433-439. (in Chinese)

[52] Z Q Liu. Ultrasonic axial stress meter with a single-chip microcomputer at its core. Industrial Instrumentation and Automation, 1993(5): 49-51. (in Chinese)

[53] L Wang. Influence of bolt shape on ultrasonic fastening stress measurement. Measurement Technique, 1999(4): 23-25. (in Chinese)

[54] Q X Pan, C Shao, D G Xiao, et al. Study of ultrasonic measurement method for bolt fastening force based on shape factor. Acta Armamentarii, 2019, 40(4): 880-888. (in Chinese)

[55] X D Chen, JT Zhu. Application research of ultrasonic technique to measure the bolt stress. China Measurement and Testing Technology, 1996(5): 5-8. (in Chinese)

[56] Z Q Liu, J N Hua, S Liang, et al. Determination of ultrasonic wave velocitystress-temperature relations in bolt materials inspection. Applied Acoustics, 1997, 16(5): 26-31. (in Chinese)

[57] B J An. Research on the experiments of electromagnetic acoustic transducer testing mechanism. Wuhan: Huazhong University of Science and Technology, 2008. (in Chinese)

[58] Z Ma. Design and development of electromagnetic ultrasonic testing instrument based on FPGA. Nanchang: Nanchang Hangkong University, 2017. (in Chinese)

[59] XY Chen. Design of electromagnetic acoustic transducers for inspection of rail base. Harbin: Harbin Institute of Technology, 2014. (in Chinese)
[60] L Hu. Design and experimental research on electromagnetic acoustic transducer. Beijing: China Academy of Machinery Science and Technology, 2013. (in Chinese)

[61] P H Xin. The research on transduction efficiency of surface wave EMAT. Harbin: Harbin Institute of Technology, 2010. (in Chinese)

[62] G Li, Y M Mo, NWu. Research and prospect on the technique for bolt axial stress measurement. Journal of Nantong University (Natural Science Edition), 2009, 8(3): 67-71. (in Chinese)

[63] L Lu, D Q Xiao, D M Lin, et al. Study on the preparation of niobate leadfree piezoelectric ceramic films by pulse laser deposition. Piezoelectrics and Acoustooptics, 2009, 31(1): 94-96, 99. (in Chinese)

[64] R B Thompson. Physical principles of measurements with EMAT transducers. Physical Acoustics, 1990, 19: 157-200.

[65] M Hirao, H Ogi. EMATs for science and industry. Springer US, 2003.

[66] Y C Zheng. Study on yielded torque-angle tightening system. Design and Manufacture of Diesel Engine, 2009, 16(2): 43-47. (in Chinese)

[67] R Cheng, K F Zou, B J Yao, et al. Research on the tightening methods of engine cylinder cover bolt. Value Engineering, 2016, 35(6): 120-121. (in Chinese)

[68] H Chu, X G Shi. Test methods for residual bolt tension by using ultrasonic technique. Journal of Netshape Forming Engineering, 2017, 9(1): 119-124. (in Chinese)

[69] J L Jia, C M Wang, TW Li, et al. Ultrasonic method research on residual tension testing of bolt with assembling to yield. Mechanical Engineering and Technology, 2018, 7(4): 245-251. (in Chinese)

\section{Submit your manuscript to a SpringerOpen ${ }^{\odot}$ journal and benefit from:}

- Convenient online submission

- Rigorous peer review

- Open access: articles freely available online

- High visibility within the field

- Retaining the copyright to your article

Submit your next manuscript at $\boldsymbol{\nabla}$ springeropen.com 